\title{
Development of Low Loss, Wide Bandwidth Hollow Core Photonic Bandgap Fibers
}

\author{
M.N. Petrovich, N.K. Baddela, N.V. Wheeler, E. Numkam, R. Slavík, D. R. Gray, J.R. Hayes, \\ J.P. Wooler, F. Poletti and D. J. Richardson \\ Optoelectronics Research Centre, University of Southampton, Highfield, Southampton, SO17 1BJ, UK \\ mnp@orc.soton.ac.uk
}

\begin{abstract}
We present a detailed overview of progress in the development of hollow-core photonic bandgap fibers with improved transmission and modal properties which demonstrates the feasibility and potential of this fiber solution for future telecom systems.

OCIS codes: (060.5295) Photonic crystal fibers; (060.2280) Fiber design and fabrication; (060.2330) Fiber optics communications
\end{abstract}

\section{Introduction}

Strong interest in radically novel types of transmission fibers is being fuelled at present by a growing concern that current transmission systems are close to their intrinsic capacity limits and will be unable to cope with the future bandwidth demands of internet traffic in a cost effective manner [1]. Spatial division multiplexing (SDM) schemes aim at realizing multiple channels in multicore or multimode fibers [2], thereby up-scaling the available capacity per fiber. A more radical approach seeks to address the fundamental limits of loss and nonlinearity of transmission fibers. Hollow core Photonic Bandgap Fibers (PBGFs) contain complex photonic crystal cladding structures which provide tight, wavelength-specific light confinement in a hollow core. As the overlap with the glass structure is only fractional, they readily offer orders-of-magnitude reduction of the optical nonlinearity, ultimate low-latency (close to the vacuum speed of light) and potential for ultra-low transmission loss. Moreover, PBGFs are inherently multimoded, which may eventually enable SDM transmission schemes, thus providing a further incentive for this technology. Despite the obvious potential advantages of PBGFs, activity in telecom applications had been very limited in the past; more recently however there has been a strong resurgence of interest in these fibers, stimulated by a number of breakthrough results. In this paper we review the substantial progress made in the last couple of years, focusing in particular on efforts to increase the transmission bandwidth, reduce the transmission loss, and control the modal properties, and discuss direction of future improvement.

\section{Widening the gap: transmission bandwidth maximization}

Whilst much emphasis is placed on loss reduction, the importance of achieving low loss over a suitably wide wavelength interval in PBGFs is not to be underestimated. The extent of the intrinsic operation window of PBGFs is determined primarily by their cladding structure (i.e. lattice geometry, pitch and air filling factor). However, the bandwidth of real fibers is often determined by resonant couplings to surface modes (SMs), whose spectral position is determined by the structure of the photonic crystal cladding/core boundary (i.e. thickness, radii and ellipticity). SMs are known to decrease - often dramatically - the transmission bandwidth of PBGFs and to increase their minimum loss. They also adversely impact other key transmission properties, such as group delay difference and birefringence, by causing large and unpredictable variations due to their extreme sensitivity to small scale structural variations of the core boundary along the fiber length. Effective suppression of SMs has been theoretically predicted and experimentally demonstrated in PBGFs based on smaller core defects (i.e. 3-cell [3] and 7-cell [4]) by designing the fiber to have an 'ideal' core boundary approximately one half as thick as the struts in the cladding with minimal distortion of the adjacent cladding holes [5]. However, this becomes substantially more challenging in structures with large core sizes and, until very recently, the bandwidth reported for 19 cell PBGFs was invariably substantially narrower than the smaller core counterparts, in spite of the potentially lower transmission loss.

We have demonstrated 19 cell PBGFs that simultaneously provide ultra-low loss and a wide transmission bandwidth [6]. Fibers were fabricated via a two-stage stack and draw technique where the core was obtained as a vacancy rather than by inserting a large core tube as is customary for these fibers. Whilst the core boundary does not conform to ideal design rules, thus introducing SMs, we have found that the spectral location of these modes can be controlled by carefully engineering the core and cladding expansion ratios (as shown in Fig. 1a) and that 3-dB transmission bandwidths well in excess of $150 \mathrm{~nm}$ (and possibly more) can be repeatably obtained in real fibers. 

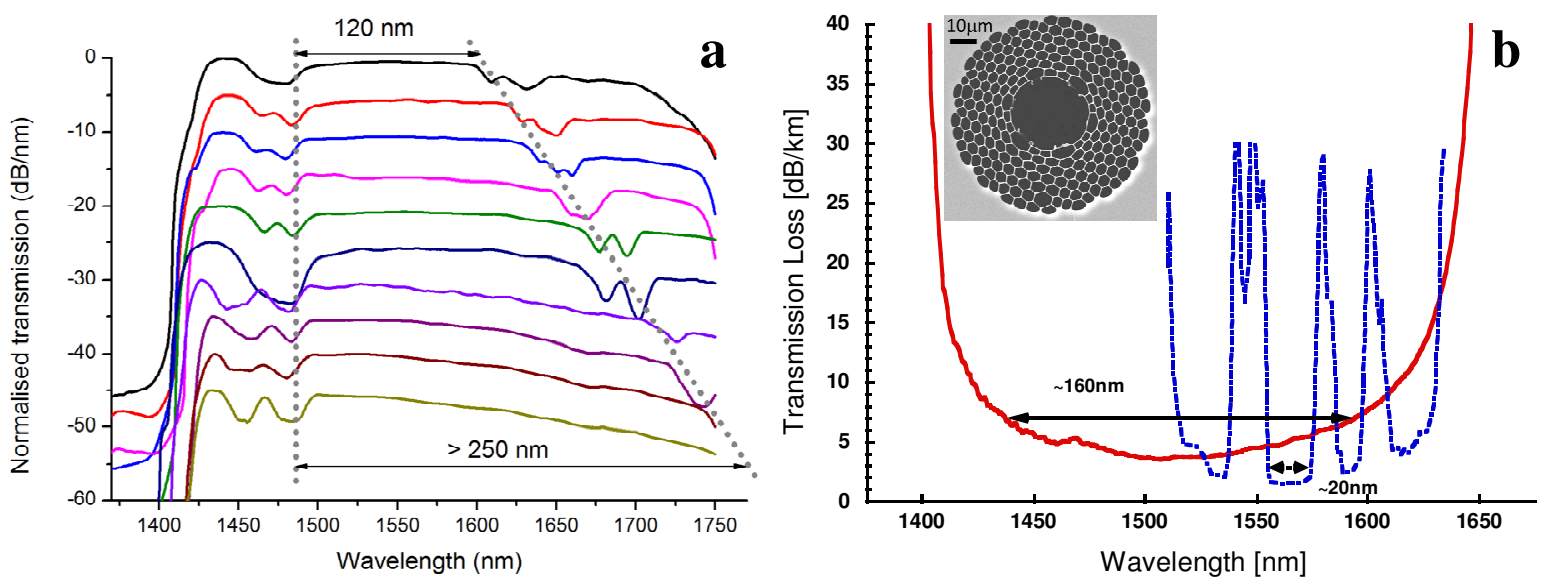

Fig. 1: (a) Bandwidth enhancement via surface mode control. (b) Attenuation of 19 cell PBGF with record combination of low loss $(3.5 \mathrm{~dB} / \mathrm{km})$ and wide bandwidth $(160 \mathrm{~nm})$. Fiber in ref [7] shown to highlight the bandwidth improvement.

Shown in Fig $1 \mathrm{~b}$ is the transmission spectrum of a 19 cell PBGF with an unprecedented combination of low loss $(3 \mathrm{~dB} / \mathrm{km})$ at $1.5 \mu \mathrm{m}$ and wide bandwidth of $160 \mathrm{~nm}$. This value is over eight times wider than the record low-loss PBGF [7], covering the combined $\mathrm{S}+\mathrm{C}+\mathrm{L}$ band, which is over $20 \mathrm{THz}$ wide.

\section{Loss Reduction Strategies}

Loss in PBGFs is dominated by optical scattering caused by nanoscale roughness at the air-glass boundaries and existing evidence points to a fundamental limit due to surface capillary waves [8]. As it is unclear whether such roughness can be effectively modified, current loss reduction strategies mainly focus on reducing the strength of the electromagnetic field at the air/glass interfaces through fiber design optimization. A straightforward way to reduce the field intensity is by increasing the core radius, $\mathrm{R}$, which is known to produce a reduction in scattering loss approximately proportional to $\mathrm{R}^{-3}$. For instance, loss in a 37 cell PBGF would be expected to have a factor of $\sim 3$ lower transmission loss as compared to an equivalent 19 cell PBGF.

Both models and existing experimental data predict the minimum loss in a PBGF to be at around 1.9-2 $\mu \mathrm{m}$. This is a consequence of the material multiphonon absorption being substantially reduced due to the low overlap (typically $\leq 0.1 \%$ ) and of the $\lambda^{-3}$ wavelength dependence of the scattering loss. The wavelength scaling predicts a factor of 2 loss reduction solely through moving the operating wavelength from 1.5 to $2 \mu \mathrm{m}$. This may eventually represent a significant enough improvement to justify the shift away from conventional telecom wavelengths. It is thus important to assess the performance of PBGFs operating at $2 \mu \mathrm{m}$, recognizing that additional loss mechanisms may be present. We successfully demonstrated PBGFs with loss as low as $4.5 \mathrm{~dB} / \mathrm{km}$ and BWs comparable to $1.5 \mu \mathrm{m}$ fibers $[9,10]$. Transmission in this spectral region can be adversely affected by absorption from atmospheric gas species such as $\mathrm{CO}_{2}$ and $\mathrm{H}_{2} \mathrm{O}$, which in principle can also arise from the fabrication process itself. We have initiated work aimed at identifying their origin along with means for their removal [9]. In particular, through simple experiments (see Fig.2a,b) we have determined that $\mathrm{CO}_{2}$, which affects the loss at about $2.0 \mu \mathrm{m}$, is likely of atmospheric origin and, most importantly, that it can be fully eliminated through fabrication improvements. We successfully fabricated $\sim \mathrm{km}$ long fibres with no evidence of $\mathrm{CO}_{2}$ absorption. It should be noted that the region of minimum loss conveniently overlaps with the operation window of Thulium doped fiber amplifiers (TDFAs), as illustrated in the plot in Fig 2c. Very recently, a proof-of-principle demonstration of amplified transmission over a 290m length of PBGF was also reported [10], providing evidence of the technological viability of data transmission in this new spectral window.

\section{Managing the Modal Content}

Ultra-low loss is only feasible in PBGF structures that are inherently multimoded. Therefore, the ability to control and manage their modal content is a key step towards their successful application for data transmission. By using a time-of-flight technique, we obtained convincing evidence (Fig. 2d) that individual modes can be selectively exited in our fibers -even using a relatively simple launch arrangement- with negligible modal cross coupling [6]. Through $\mathrm{LP}_{01}$-optimized launch and by applying a spatial filter at the output end, we demonstrated that our fibers can be effectively operated as single-mode with better than 30dB HOM suppression (Fig. 2e). Further evidence was 
provided by full transmission system tests showing negligible penalty [11]; furthermore, $\sim 1.54 \mu \mathrm{s} / \mathrm{km}$ lower latency as compared to conventional fibers was also demonstrated [11]. The current capacity record of data transmission stands at $1.48 \mathrm{Tbits} / \mathrm{s}(37 \mathrm{x} 40 \mathrm{Gbit} / \mathrm{sec}$ channels spanning the $\mathrm{C}$-band) obtained over a 250m length of PBGF. Further capacity improvement is expected by employing more advanced modulation formats and coherent detection.
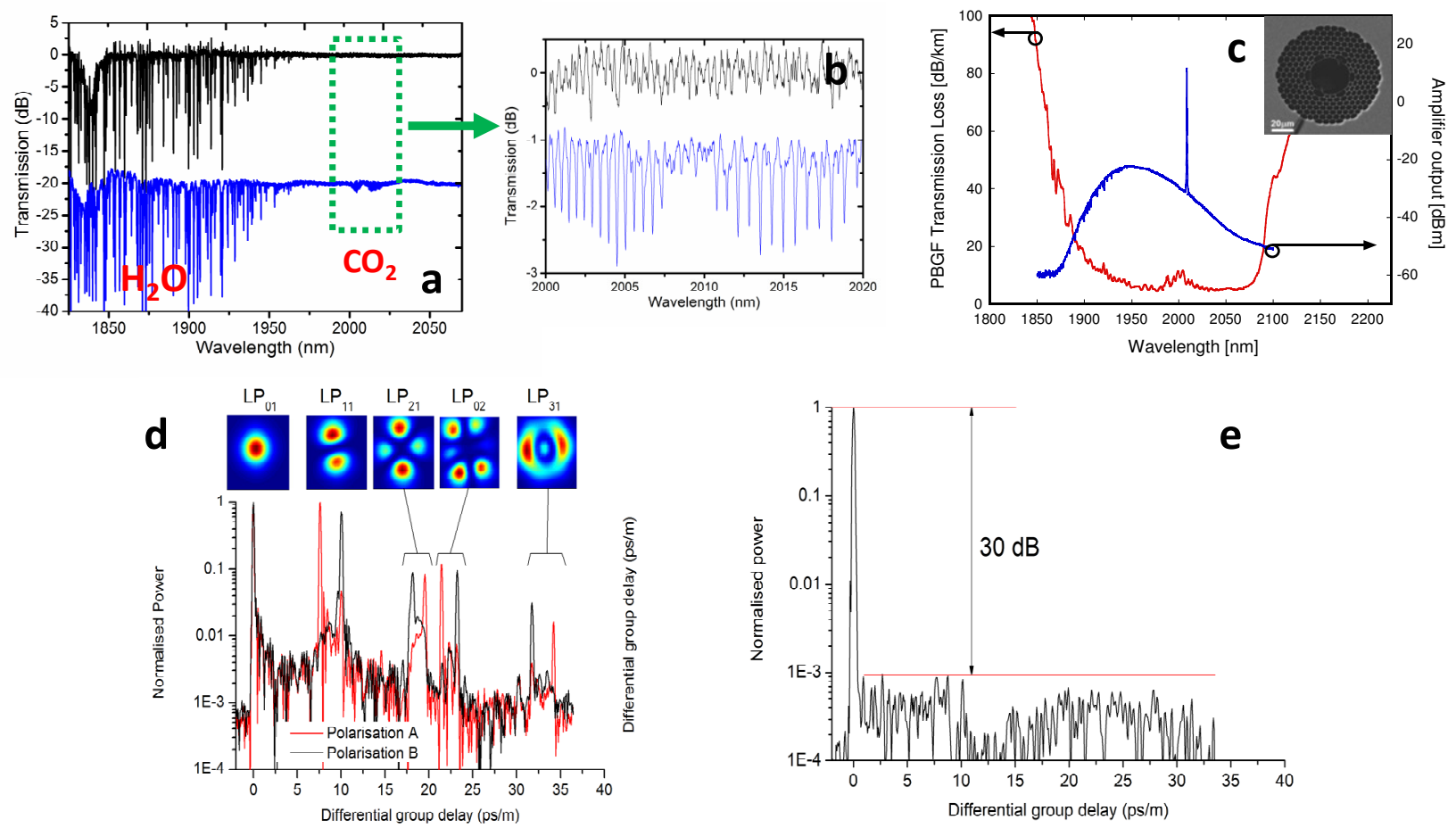

Fig. 2: (a) Transmission spectra of PBGFs at around $2 \mu \mathrm{m}$ (119m length) highlighting $\mathrm{CO}_{2}$ and $\mathrm{H}_{2} \mathrm{O}$ absorption lines and the effect of purging with dry nitrogen particularly on $\mathrm{CO}_{2}$ (b); (c) Attenuation of a $4.5 \mathrm{~dB} / \mathrm{km}$ PBGF at $2 \mu \mathrm{m}$ highlighting match with

TDFA gain BW; (d) Selective modal excitation in a PBGF at $1.55 \mu \mathrm{m}$ (e) effectively single-mode operation of the same.

\section{Conclusions}

PBGFs are the subject of a resurgent interest due to their low nonlinearity and low latency. We have provided an overview of recent fiber improvements which have enabled landmark proof-of-principle transmission system experiments. Low loss, wide bandwidth PBGFs with negligible modal cross coupling have been demonstrated and further improvements, in particular loss reduction, appear feasible. Although many difficult challenges are yet to be addressed, the results obtained so far illustrate the potential of this radically new fiber solution for next generation transmission systems and are a stepping stone in the assessment of their technological viability. This work was supported by the EU 7th Framework Programme under grant agreement 228033 (MODE-GAP) and by the UK EPSRC through grant EP/H02607X/1.

\section{References}

[1] E. Desurvire, et al., "Science and technology challenges in XXIst century optical communications", Comptes Rendus Physique 12 (2011) 387

[2] R Ryf, et al., "Space division multiplexing over 10km of three moded fiber using coherent 6x6 MIMO processing", Proc. OFC2011, PDPB10

[3] M. N. Petrovich et al., "Robustly single mode hollow core photonic bandgap fiber", Opt Express 16 (2008) 4337

[4] R. Amezcua-Correa, et al., "Control of surface modes in low loss hollow-core photonic bandgap fibers," Opt. Express 16 (2008) 1142.

[5] R. Amezcua-Correa, et al., "Design of 7 and 19 cells core air-guiding photonic crystal fibers for low-loss, wide bandwidth and dispersion controlled operation," Opt. Express, 15(2007) 17577

[6] Wheeler, et al. "Wide-bandwidth, low-loss, 19-cell hollow core photonic band gap fiber and its potential for low latency data transmission," Proc. OFC 2011, PDP5A.2.

[7] B.J. Mangan, et al., "Low loss (1.7 dB/km) hollow core photonic bandgap fiber", Proc. OFC 2004, PD24

[8] P. J. Roberts, et al., " Ultimate low loss of hollow-core photonic crystal fibres", Opt. Express, 13 (2005) 236.

[9] Wheeler, et al. " Gas Absorption between 1.8 and $2.1 \mu \mathrm{m}$ in low loss (5.2 dB/km) HC-PBGF", Proc. CLEO 2012, paper CM3N

[10]Petrovich et al., "First demonstration of $2 \mu \mathrm{m}$ data transmission in a Low-loss hollow core photonic bandgap fiber", Proc. ECOC 2012 PDPTh3.A.5

[11]Slavik, et al., "1.45 Tbit/s, Low Latency Data Transmission through a 19-cell hollow core photonic band gap fibre", Proc. ECOC 2012 Mo.2.F 